\title{
Gauge boson mass generation-without Higgs-in the scalar strong interaction hadron theory
}

\section{F. C. Hoh}

Dragarbrunnsg, 55C, Uppsala, Sweden; hoh@telia.com

Received 6 December 2009; revised 23 December 2009; accepted 5 January 2010.

\section{ABSTRACT}

It is shown that the gauge boson mass is naturally generated-without Higgs-in the pion beta decay using the scalar strong interaction hadron theory. This mass generation is made possible by the presence of relative time between quarks in the pion in a fully Lorentz covariant formalism.

Keywords: No Higgs; Gauge Boson Mass; Scalar Strong Interaction

The nonobservation of Higgs, needed in the standard model [1], has led to various supersymmetry models that have no experimental support. This gauge boson mass generation problem is resolved here in the scalar strong interaction hadron theory (SSI) [2,3], an alternative to low energy QCD. The equations of motion of mesons, not yet quantized, read $[2,3]$.

$$
\begin{gathered}
\partial_{I}^{a \dot{b}} \partial_{I I}^{f \dot{e}} x_{(p r) b j}\left(x_{I}, x_{I I}\right)-\left(M_{m p r}^{2}-\Phi_{m}\left(x_{I}, x_{I I}\right)\right) \\
\psi_{(p r)}^{a \dot{e}}\left(x_{I}, x_{I I}\right)=0 \\
\partial_{I b c} \partial_{I I e d d} \psi_{(p r)}^{c \dot{e}}\left(x_{I}, x_{I I}\right)-\left(M_{m p r}^{2}-\Phi_{m}\left(x_{I}, x_{I I}\right)\right) \\
x_{(p r) b \dot{b}}\left(x_{I}, x_{I I}\right)=0, \quad M_{m p r}=\frac{1}{2}\left(m_{p}+m_{r}\right) \\
\square_{I} \square_{I I} \Phi_{m}\left(x_{I}, x_{I I}\right)= \\
-\frac{g_{s}^{4}}{4}\left(\psi^{b \dot{b}}\left(x_{I}, x_{I I}\right) \chi_{\dot{a} b}^{*}\left(x_{I}, x_{I I}\right)+\psi^{* a \dot{b}}\left(x_{I}, x_{I I}\right) \chi_{b a}\left(x_{I}, x_{I I}\right)\right)
\end{gathered}
$$

Here, $x_{I}$ and $x_{I I}$ are the quark and antiquark coordinates, $\partial_{I}=\partial / \partial x_{I}, \partial_{I I}=\partial / \partial x_{I I}, \chi$ and $\psi$ are the meson wave functions with the spinor indices $a, b, \ldots$, undotted and dotted, running from 1 to $2, \Phi_{m}$ the scalar interquark potential, $g_{s}$ the strong quark charge, $m_{p}$ and $m_{r}$ the quark masses, and $p, r$ the quark flavors ( 1 for $u$ and 2 for $d$ quark). An epistemological background of this theory has been published earlier this year [4]. Eqs.1-3 have been rather successfully applied to confinement and meson spectra [5] and some basic meson decays [6-9].

In these references, the transformation

$$
x^{\mu}=x_{I I}^{\mu}-x_{I}^{\mu}, \quad X^{\mu}=\left(1-a_{m}\right) x_{I}^{\mu}+a_{m} x_{I I}^{\mu}, \quad a_{m}=1 / 2
$$

has been made. The relative space time $x^{\mu}=\left(x^{0}, \underline{x}\right)$ are hidden variables [4] reflecting the fact that no free quarks exists. Generation of gauge boson mass without Higgs is shown here by the example of pion beta decay $\pi^{+} \rightarrow \pi^{0} e^{+} v_{e}$. Formally, this requires a field theoretical treatment but here attempt is made to describe such decays on the quantum mechanical level, analogous to some semiclassical treatments of radiation in quantum mechanics. The justification is that the energies involved are low so that field-theoretical effects such as vacuum polarization are small, just like that analogous effects are small in QED at low energies.

The starting point is the total action [10]

$$
\begin{aligned}
& S_{T}=S_{G B}+S_{L l}+S_{L r}+S_{L m}+S_{m} \\
& S_{G B}=-\frac{1}{4} \int d^{4} X \sum_{l=1}^{3} G_{l}^{\mu v} G_{l \mu v} \\
& G_{l}^{\mu \nu}(X)=\partial_{X}^{\mu} W_{l}^{v}-\partial_{X}^{v} W_{l}^{\mu}-\varepsilon_{j k l} g W_{j}^{\mu} W_{k}^{v} \\
& S_{L r}=-i \frac{1}{4} \int d^{4} X \chi_{L a} \partial_{X}^{a \dot{b}} \chi_{L \dot{b}}+\text { c.c. }
\end{aligned}
$$

$$
\begin{gathered}
S_{L l}=-\frac{i}{4} \int d^{4} X\left(\psi_{v L}^{\dot{a}}, \psi_{L}^{\dot{a}}\right)\left[\partial_{X a b}+\frac{i}{2} g\left(\begin{array}{cc}
W_{3} & \sqrt{2} W^{-} \\
\sqrt{2} W^{+} & -W_{3}
\end{array}\right)_{a b}\right]\left(\begin{array}{l}
\psi_{v L}^{b} \\
\psi_{L}^{b}
\end{array}\right)+c c . \\
S_{L m}=-\frac{1}{2} \int d^{4} X m_{L}\left(\psi_{L}^{\dot{a}} \chi_{L \dot{a}}+\text { c.c. }\right)
\end{gathered}
$$

where $S_{G B}$ is the action for the gauge boson fields $W^{+}, W$ and $W_{3}$ and $S_{L}$ the $\mathrm{SU}(2)$ part of the lepton action in the standard model. $S_{m}$ is the SSI meson action generalized to include $\mathrm{SU}(2)$ gauge fields 


$$
\begin{aligned}
& S_{m}=\frac{1}{2} \int d^{4} X d^{4} x\left(\begin{array}{l}
-\left(D_{I p s}^{a \dot{b}+} \chi_{(r p) \dot{e} a}^{+}\right)\left(D_{I I s q}^{f \dot{e}} \chi_{(q r) \dot{b j}}\right)-\left(D_{I p s \dot{b}}^{+} \psi_{(r p)}^{+e \dot{a}}\right)\left(D_{I I s q \dot{j e}} \psi_{(q r)}^{b \dot{f}}\right)- \\
\chi_{(r p) \dot{e} a}^{+}\left(M_{m p r}^{2}-\Phi_{m}\right) \psi_{(p r)}^{a \dot{e}}-\psi_{(r p)}^{+a \dot{e}}\left(M_{m p r}^{2}-\Phi_{m}\right) \chi_{(p r) \dot{e} a}+c . c .
\end{array}\right) \\
& \partial_{I}^{a \dot{b}}=\frac{1}{2} \partial_{X}^{a \dot{b}}-\partial^{a \dot{b}} \rightarrow D_{I p s}^{a \dot{b}}=\frac{1}{2}\left(\partial_{X}^{a \dot{b}} \delta_{p s}+i \frac{1}{2} g\left(\sigma_{l}\right)_{p s} W_{l}^{a \dot{b}}(X)\right)-\partial^{a \dot{b}} \delta_{p s} \\
& =\partial_{I}^{a \dot{b}} \delta_{p s}+i \frac{1}{4} g\left(\sigma_{l}\right)_{p s} W_{l}^{a \dot{b}}(X) \\
& \left(\sigma_{l}\right)_{p s} W_{l}^{a \dot{b}}(X)=\left(\begin{array}{cc}
W_{3} & \sqrt{2} W^{-} \\
\sqrt{2} W^{+} & -W_{3}
\end{array}\right)^{a \dot{b}}, \sqrt{2} W^{ \pm}=W_{1} \pm i W_{2}
\end{aligned}
$$

The superscript ${ }^{+}$in Eq.11 denotes hermitian conjugation. Lorentz and gauge invariance of Eqs.4-10 has been established in $[2,3]$. Here,

$$
\psi_{(p r)} \rightarrow\left(\begin{array}{cc}
\frac{1}{\sqrt{2}} \psi_{(\pi 0)} & \psi_{(\pi+)} \\
\psi_{(\pi-)} & -\frac{1}{\sqrt{2}} \psi_{(\pi 0)}
\end{array}\right), \quad \psi \rightarrow \chi
$$

Variation of Eq.11 with respect to $\chi^{+}$and $\psi^{+}$, with boundary conditions specified in [12], yields

$$
\begin{aligned}
& D_{I p s}^{a \dot{b}} D_{I I s q}^{f e} \chi_{(q r) \dot{b f}}-\left(M_{m p r}^{2}-\Phi_{m}\right) \psi_{(p r)}^{a \dot{e}}=0 \\
& D_{I p s \dot{c} a} D_{I I s q \dot{d} d} \psi_{(q r)}^{a \dot{e}}-\left(M_{m p r}^{2}-\Phi_{m}\right) \chi_{(p r) \dot{c} d}=0
\end{aligned}
$$

In the limit of $g \rightarrow 0$, Eq.15 and Eq.16 reproduce Eqs. 1 and 2 together with subsidiary conditions, arising from the c.c term in Eq.11, that are satisfied at least for plane wave $W$, which refers to $W^{+}$here.

Following [8], let the meson wave functions be perturbed:

$$
\begin{aligned}
& \psi_{(p r)}^{a \dot{b}}(X, x)=\left(a_{p r}+a_{p r}^{(1)}\left(X^{0}\right)\right) \times \\
& \left(\delta^{a \dot{b}} \psi_{0(p r) K}(\underline{x})-\underline{\sigma}^{a \dot{b}} \underline{\psi}_{(p r) K}(\underline{x})\right) \exp \left(-i E_{p r K} X^{0}+i \underline{K}_{p r} \underline{X}\right) \\
& =\psi_{0(p r)}^{a \dot{b}}(X, x)+\psi_{1(p r)}^{a \dot{b}}(X, x)=\left(1+\frac{a_{p r}^{(1)}\left(X^{0}\right)}{a_{p r}}\right) \\
& \psi_{0(p r)}^{a \dot{b}}(X, x), \quad \psi \rightarrow \chi
\end{aligned}
$$

Here, the index 1 denotes a first order quantity, $a_{p r}$ is unity here but is in a quantized case to be elevated to an annihilation operator annihilating a initial meson with flavor $p r$. Its complex conjugate $a_{r s}^{*}$ is also unity and is elevated to a creation operator creating a final state with flavor $r s . \quad a_{p s}^{(1)}\left(X^{0}\right)$ is a small amplitude that varies slowly with time and, in the quantized case, becomes an operator that "slowly" transforms the same initial state meson to some virtual intermediate vacuum state. It is zero at $X^{0}=-\infty$. $a_{r s}^{(1)^{*}}\left(X^{0}\right)$ is the complex conjugate of $a_{p s}^{(1)}\left(X^{0}\right)$ and, in the quantized case, becomes an operator that "slowly" creates the same final state as that cre- ated by $a_{r s}^{*}$. The subscripts $p r$ have also been attached to $E_{K}$ and $\underline{K}$ of the meson. It has been shown that the last of Eq.4, required by decay applications [8], leads to that Eq.17 is independent of the relative time $x^{0}$.

The terms in the actions can now be grouped in powers of the small parameter $g$. Only the lowest order and independent quantities are listed in the two alternatives below [3]:

First order: $\quad g, \quad a_{p r}^{(1)}\left(X^{0}\right), \partial_{X}$ in $S_{G B}, \psi_{L}^{a} \psi_{L}^{b}, \quad \chi_{L a} \chi_{L \dot{b}}$

Firstorder: $\quad g, \quad W, \partial_{X}$ in $S_{G B}, \psi_{L}, \chi_{L}$

Second order : $\quad a_{p r}^{(1)}\left(X^{0}\right)$

Insert Eq.17 into Eq.15 and Eq.16, multiply Eq.15 by $\chi_{0 \dot{a} a(r p)}^{*}$ and Eq.16 $\psi_{0(r p)}^{* d \dot{c}}$, add them together, and integrate over $X$ and $x$. The first order quantities read

$$
S_{m d}^{\prime}=S_{m s}^{\prime}
$$

Here, $S_{m d}^{\prime}$ is linear in the first order quantity $a_{p s}^{(1)}\left(X^{0}\right)$,

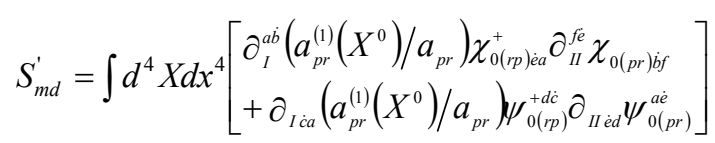

The source part $S_{m s}^{\prime}$ of the first order terms contains the $g W$ terms in Eq.11, ignoring the c.c. term there,

$$
S_{m s}^{\prime}=-\int d^{4} X d x^{4} \frac{i g}{4}\left\{\begin{array}{l}
\chi_{0(r p) \dot{e} a}^{+}\left(\sigma_{l}\right)_{p s} W_{l}^{a \dot{a}}\left(\partial_{I I}^{f e} \chi_{0(s r) b \dot{b j}}\right) \\
-\left(\partial_{I}^{a \dot{b}} \chi_{0(r p) \dot{e} a}^{+}\right)\left(\sigma_{l}\right)_{p s} W_{l}^{f e} \chi_{0(s r) b \dot{b j}} \\
+\psi_{0(r p)}^{+d \dot{c}}\left(\sigma_{l}\right)_{p s} W_{l c a}\left(\partial_{I I \dot{e} d} \psi_{0(s r)}^{a \dot{e}}\right) \\
-\left(\partial_{I \dot{c} a} \psi_{0(r p)}^{+d \dot{c}}\right)\left(\sigma_{l}\right)_{p s} W_{l \dot{e} d}^{a \dot{e}} \psi_{0(s r)}^{a \dot{e}}+R_{s}
\end{array}\right\}
$$

where $R_{S}$ is a surface term [3] which vanishes upon integration in Eq.29 below. As a rudimentary quantization procedure, let

$$
|i\rangle=\left|P_{i(12)}\left(E_{12}\right)\right\rangle, \quad\langle f|=\left\langle P_{f(11-22)}\left(K_{f(11-22)}^{\mu}\right), W\right|
$$


where $P_{i(12)}$ represents the initial $\pi^{+}, P_{f(11-22)}$ the final state $\pi^{0}$ and $W$ the intermediary boson. The subscript $K$ in Eq.17 is zero for the initial $\pi^{+}$and is suppressed.

$a_{p r}$ in Eq.17 is now elevated to become an annihilation operator according to

$$
a_{p r}\left|P_{i(p r)}\left(E_{p r}\right)\right\rangle=|0\rangle
$$

Similarly, $a_{r s}^{*}$ is interpreted as a equivalent creation operator acting on $\mid 0>$ or an annihilation operator acting on $<f \mid$. Along these lines, the decay amplitude has been defined as [3]

$$
S_{f i}=\left\langle f \mid a_{r p}^{+} a_{p r}^{(1)}\left(X^{0} \rightarrow \infty\right) \cdot i\right\rangle
$$

The zeroth order wave functions for a pseudoscalar meson at rest are obtained by solving Eqs.1-3 using Eq.17 and are [3,7]

$$
\begin{gathered}
\psi_{o(p r)}^{a b}(X, x)=\delta^{a b} \psi_{o(p r)}(r) \exp \left(-i E_{p r} X^{0}\right), \quad \psi \rightarrow \chi \\
\psi_{o(p r)}(r)=-\chi_{o(p r)}(r)=\psi_{0}(r)=\sqrt{\frac{d_{m}^{3}}{8 \pi \Omega}} \exp \left(-d_{m} r / 2\right), \\
d_{m}=0.864 \mathrm{Gev}
\end{gathered}
$$

Inserting these into Eq.17 and Eq.21 and place Eq.20 between $<f \mid$ and $\mid i>$ yields:

$$
S_{f i}=i \frac{2}{E_{p r} \tau_{0}}\left\langle f\left|S_{m s}^{\prime}\right| i\right\rangle, \quad \tau_{o}=\int d x^{0} \rightarrow \infty
$$

where $E_{p r}$ is the mass of the initial $\pi^{+}$. With Eq.22 and Eqs.26 and 27, Eq.28 becomes

$$
\begin{gathered}
S_{f i}=-\frac{i g}{4 \Omega E_{\pi+}} \int d^{4} X \exp \left(i\left(E_{\pi 0}-E_{\pi+}\right) X^{0}-i \underline{K}_{\pi 0} \underline{X}\right) \\
\times\left[\left(E_{\pi+}+E_{\pi 0}\right) W^{0+}(X)+2 \underline{K}_{\pi 0} \underline{W}^{+}(X)\right], \\
\quad \Omega=\int d^{3} X \rightarrow \infty
\end{gathered}
$$

This result can also be obtained starting from either Eq.15 or Eq.16, without the addition operation mentioned below Eq.19.

Variation of Eqs.6 and 7 with respect to $W^{-a \dot{b}}$ defined in Eq.13 yields

$$
\delta S_{G B} / \delta W^{-a \dot{b}}=\frac{1}{2} \square W_{\dot{b} a}^{+}-\frac{1}{4} \partial_{X \dot{x} a}\left(\partial_{X}^{c \dot{d}} W_{\dot{d c}}^{+}\right)+\frac{1}{2} g^{2} V_{C \dot{b} a}\left(W^{+}\right)
$$

where $V_{C}$ is trilinear in $W$. Variation of the same order part of Eq.11 yields

$$
\begin{aligned}
& \delta S_{m} / \delta W^{-a b} \\
& =-\frac{g^{2}}{16} \int d^{4} x W^{+f e}\left[\begin{array}{l}
\left(\chi_{0(12) \dot{e a}}^{*} \chi_{0(12) \dot{b j}}+\chi_{0(21) \dot{b j}}^{*} \chi_{0(21) \dot{e} a}+\chi_{0}^{*} \leftrightarrow \chi_{0}\right) \\
+\left(\psi_{0(21) \dot{e} a} \psi_{0(21) \dot{b} f}^{*}+\psi_{0(12) \dot{b} f} \psi_{0(12) \dot{e} a}^{*}+\psi_{0}^{*} \leftrightarrow \psi_{0}\right)
\end{array}\right]
\end{aligned}
$$

Inserting Eqs.26 and 27 into Eq.31 yields

$$
\delta S_{m} / \delta W^{-a \dot{b}}=-\frac{g^{2}}{2} \frac{\tau_{0}}{\Omega} W^{+b \dot{a}}
$$

The same variation applied to Eqs.8-10 yields

$$
\delta S_{L} / \delta W^{-a b}=\frac{g}{2 \sqrt{2}} \psi_{v L b} \psi_{L a}
$$

where $L$ on the right side refers to $e^{+}$. With Eqs.30-33, variation of Eq.5 with respect to $W^{-a \dot{b}}$ gives

$$
\begin{gathered}
\square W_{\dot{b} a}^{+}-\frac{1}{2} \partial_{X b \dot{ }}\left(\partial_{X}^{c \dot{d}} W_{\dot{d} c}^{+}\right)+g^{2} V_{c \dot{a} a}\left(W^{+}\right)-M_{W}^{2} W^{+b \dot{a}}=-\frac{g}{\sqrt{2}} \psi_{v i b} \psi_{L a} \\
M_{W}^{2}=g^{2} \frac{\tau_{0}}{\Omega}
\end{gathered}
$$

$M_{W}$ is the mass of the charged gauge boson [7] and its square the ratio of an integral over the relative time $x^{0}$ between the quarks of the pion and the normalization volume $\Omega$ of the pion wave function. By the last of Eq.28 and Eq.29, this ratio is $\infty / \infty=$ finite. The pions here also play the role of the Higgs in the standard model. That Higgs boson is not needed to generate $M_{W}$ was first shown in [12].

Contracting Eq.34 by $\delta^{a \dot{b}}$ and $\underline{\sigma}^{a \dot{b}}$ yields

$$
\begin{gathered}
-\frac{\partial}{\partial X^{0}}\left(\frac{\partial}{\partial \underline{X}} \underline{W}^{+}\right)+g^{2} V_{C}^{0}\left(W^{+}\right)-M_{W}^{2} W^{0+}=-\frac{g}{2 \sqrt{2}} \psi_{v L \dot{a}} \psi_{L a} \\
\square \underline{W^{+}}+\frac{\partial}{\partial \underline{X}}\left(\frac{\partial}{\partial X^{0}} W^{0+}+\frac{\partial}{\partial \underline{X}} \underline{W}^{+}\right) \\
+g^{2} \underline{V}_{C}\left(W^{+}\right)+M_{W}^{2} \underline{W}^{+}=-\frac{g}{2 \sqrt{2}} \underline{\sigma}^{a \dot{b}} \psi_{v l b} \psi_{L a}
\end{gathered}
$$

Choose the gauge [3] to be the Coulomb type

$$
(\partial / \partial \underline{X}) \underline{W}^{+}=0
$$

Further, the ordering Eqs.18 and 19 adopted relegates the nonlinear $g^{2} V_{C}$ terms in Eqs.36 and $\mathbf{3 7}$ to higher order. In the absence of the lepton source terms on the right of Eqs.36 and 37, it yields to lowest order

$$
\begin{aligned}
& W^{0+}=0 \\
& \square \underline{W}^{+}+M_{W}^{2} \underline{W}^{+}=0
\end{aligned}
$$

$\underline{W}^{+}$is identified with the observed charged gauge boson $\underline{W}[1]$ with the mass

$$
M_{W}=80.42 \mathrm{Gev}
$$

The time component $W^{0+}$ associated with $\underline{W}^{+}$in Eqs.39 and 40 vanishes in agreement with the nonobservation of such a singlet charged gauge boson $W^{0+}$ accompanying the observed triplet $\underline{W}^{+}$. If Higgs boson were used to generate the gauge boson mass, such a singlet $W^{0+}$ with same mass Eq.41 should also be seen, contrary to observation. 
If the Lorentz gauge

$$
\partial_{X}^{c d} W_{d c}^{+}=0
$$

were employed, Eq.40 remains unchanged and Eq.39 becomes

$$
\square W^{0+}-M_{W}^{2} W^{0+}=0
$$

This implies that $W^{0+}$ has an imaginary mass of Eq.41 and therefore must vanish and $\mathbf{E q . 3 9}$ remains in effect valid.

The energy and momentum of the virtual gauge boson in Eqs.36 and $\mathbf{3 7}$ are determined by those of the lepton pair and are small and can be dropped next to the mass terms. Hence, Eqs.36 and $\mathbf{3 7}$ reduces to

$$
\begin{gathered}
M_{W}^{2} W^{0+}=\frac{g}{2 \sqrt{2}} \psi_{L a}^{(-)} \psi_{v L \dot{a}}^{(+)} \\
M_{W}^{2} \underline{W}^{+}=-\frac{g}{2 \sqrt{2}} \underline{\sigma}^{b \dot{a}} \psi_{\nu \grave{L}}^{(+)} \psi_{L b}^{(-)}
\end{gathered}
$$

While the triplet $\underline{W}^{+}$can exist freely and hence be seen, as is shown in Eq.40, it can also be a virtual intermediate state in Eq.45. On the other hand, the singlet $W^{0+}$ cannot be observed by Eq.39, but can only be a charged, virtual intermediate singlet as is seen in Eq.44. These results are due to that the signs of the $M_{W}^{2}$ terms in Eqs.36 and $\mathbf{3 7}$ are different, which in its turn stems from that the meson wave functions Eqs.26 and 27 are not scalar but the time component of a four vector in SSI. In pseudoscalar meson decays, only the virtual $W^{0+}$ enters.

Because Eqs.26 and $\mathbf{2 7}$ are independent of flavor, any pseudoscalar meson can generate the same $M_{W}$. When the above treatment is generalized to account for kaon decay [3], $M_{W}$ is unaltered and the neutral gauge boson mass becomes $M_{Z}=M_{W} / \cos$ (Weinberg angle) $=91.02 \mathrm{Gev}$. Decay of the $\underline{W}^{+}$boson into a lepton pair is the same as that in the standard model. Inserting Eqs.44 and $\mathbf{4 5}$ into Eq.29 leads to a pion beta decay amplitude $[3,6]$ that is $\left(E_{\pi 0} / E_{\pi+}\right)^{1 / 2} \cong 1$ times that of the literature [11] assuming conserved vector currents.

The value $M_{W}=\infty / \infty=$ finite cannot and should not be determined in the present theory so far. If $M_{W}$ were somehow obtained from some data, it implies a test of the well-established Fermi constant with far reaching consequences. This is due to that Fermi constant is proportional to $M_{W}^{-2}$ and is hence also is a ratio $\infty / \infty=$ finite.

\section{REFERENCES}

[1] Amsler, C., et al. (2008) Particle data group. Physics Letters B, 667(1-5), 1-1340.

[2] Hoh, F.C. (1993) Spinor strong interaction model for meson spectra. International Journal of Theoretical Physics, 32(7), 1111-1133.

[3] Hoh, F.C. (2009) Scalar strong interaction hadron theory. http://web.telia.com/ u80001955

[4] Hoh, F.C. (2007) Epistemological and historical implications for elementary particle physics. International Journal of Theoretical Physics, 46(2), 269-299.

[5] Hoh, F.C. (1996) Meson classification and spectra in the spinor strong interaction theory. Journal of Physics G, 22(1), 85-98.

[6] Hoh, F.C. (1998) Weinberg angle and pion beta decay in the spinor strong interaction theory. International Journal of Theoretical Physics, 37(6), 1693-1705.

[7] Hoh, F.C. (1999) Normalization in the spinor strong interaction theory and strong decay of vector meson $\mathrm{V} \rightarrow \mathrm{PP}$. International Journal of Theoretical Physics, 38(10), 2617-2645.

[8] Hoh, F.C. (1999) Radiative decay of vector meson $\mathrm{V} \rightarrow \mathrm{P} \gamma$ in the spinor strong interaction theory. International Journal of Theoretical Physics, 38(10), 2647-2664.

[9] Hoh, F.C. (2000) $\pi^{0} \rightarrow \gamma \gamma$ in the spinor strong interaction theory. International Journal of Theoretical Physics, 39, 1069.

[10] Hoh, F.C. (1997) Meson-lepton interaction in the spinor strong interaction theory. International Journal of Theoretical Physics, 36(2), 509-531.

[11] Källén, G. (1964) Elementary particle physics. AddisonWesley, Boston.

[12] Hoh, F.C. (1994) Gauge invariance and quantozation of the spinor strong interaction model. International Journal of Modern Physics A, 9(3), 365-381. 